\title{
Sincronização de ciclos econômicos na América Latina: crises e determinantes*
}

\author{
Economic cycles synchronization in Latin America: \\ downturns and causes
}

\author{
Renata Lemos Lima e João Frois Caldeira**
}

\begin{abstract}
Resumo: Este artigo examina a sincronização dos ciclos econômicos entre quatro economias da América Latina durante o período de 1968 a 2016. Usando um modelo DCC-GARCH combinado com regressões em painel, estimou-se as correlações dinâmicas entre as quatro economias e investigou-se os efeitos de crises domésticas e internacionais na sincronização de ciclos desses países e os determinantes dessa sincronização. Os resultados sugerem que a sincronização aumenta em períodos de crises internacionais e diminui em crises domésticas enfrentadas por algum dos países analisados. Entre os determinantes, o comércio bilateral é o principal responsável pela sincronização de ciclos de negócios dos países latino-americanos.
\end{abstract}

Palavras-chave: Ciclos econômicos. Sincronização. Modelo DCC-GARCH. América Latina

\begin{abstract}
This paper examines the synchronization of economic cycles between four economies in Latin America during the period from 1968 to 2016. Using a DCC-GARCH model combined with panel regressions, we estimate the dynamic correlations between the four economies and investigate the effects of domestic and international crises on the synchronization of cycles in these countries and the determinants of this synchronization. The results suggest that synchronization increases in periods of international crises and decreases in domestic crises faced by any of the countries analysed. Among the determinants, bilateral trade is the main responsible for the synchronization of business cycles of the Latin American countries.
\end{abstract}

Keywords: Economic cycles. Synchronization. DCC-GARCH model. Latin America

JEL: E32. F40

\footnotetext{
* Submissão: 19/02/2020 | Aprovação: 17/06/2020 | DOI: 10.5380/re.v42i79.71821

** Respectivamente: (1) Doutoranda em Economia Aplicada pela Universidade Federal do Rio Grande do Sul (UFRGS) | ORCID: 0000-0002-9872-4474 | E-mail: renatalemos87@gmail.com | (2) Professor do Departamento de Economia da Universidade Federal de Santa Catarina (UFSC) |ORCID: 0000-0002-07230461 | E-mail: emaildocaldeira@gmail.com | O autor agradece o suporte financeiro da Bolsa de Produtividade em Pesquisa do CNPq.
} 


\section{Introdução}

Os ciclos econômicos mostram-se um importante tópico de discussão, especialmente em períodos de grandes flutuações em variáveis agregadas dos países, onde o objetivo é o de entender como ocorrem essas flutuações e suas consequências para a economia. Mudanças nos preços, crédito e emprego tendem a afetar a conduta dos indivíduos e das firmas no ambiente econômico, gerando comportamentos cíclicos nas principais séries econômicas. Os formuladores de políticas buscam entender estes comportamentos para definir suas políticas, uma vez que estas têm diferentes efeitos dependendo da fase do ciclo em que a economia se encontra.

Do mesmo modo, o fenômeno da globalização, ou o aumento da integração econômica, torna as barreiras econômico-financeiras cada vez menores entre países. Se por um lado, beneficia-se do aumento do comércio e do fluxo de capitais, por outro, os países tornam-se mais suscetíveis à propagação de crises econômicas e financeiras. Assim, busca-se entender o comportamento sincronizado da economia de diferentes países, ou por que alguns países são mais sincronizados do que outros. As principais explicações para ciclos econômicos similares são a integração comercial e financeira entre as economias. Tanto a integração comercial quanto a financeira possuem potenciais efeitos diretos e indiretos na sincronização dos ciclos de negócios, mas com impacto total ambíguo.

A sincronização de ciclos econômicos exerce papel fundamental em regiões com união monetária, como no caso da zona do euro. Contudo, ainda que não haja integração monetária, a magnitude da sincronização pode ter importantes implicações na condução da política macroeconômica. Em casos de alto grau de sincronização de ciclos, políticas de cooperação podem ser necessárias para a estabilização das flutuações econômicas da região.

Na América Latina, o processo de abertura econômica iniciou na década de 1990, com a redução de barreiras comerciais e financeiras e consequente aumento de transações financeiras e da participação de bancos estrangeiros nos sistemas bancários nacionais. A integração econômica na região é caracterizada por acordos comercias, como Mercosul e demais acordos bilaterais, com Brasil e México se destacando como as maiores economias e com maior poder de influência nos países vizinhos. Considerando as ligações financeiras e comerciais entre esses países, é esperado que haja algum sincronismo das atividades econômicas dos mesmos. 
Posta a importância do tema, o objetivo deste trabalho é analisar o padrão de correlação e de sincronização entre as principais economias da América Latina: Argentina, Brasil, Chile e México. Buscou-se analisar qual o padrão de sincronização dos países em períodos de crises internacionais e domésticas e os determinantes da sincronização entre esses países. Dentre esses determinantes, optou-se por verificar o papel de uma possível coordenação de política monetária e fiscal, além do impacto do comércio e fluxo de investimento bilateral na sincronização. Para alcançar o objetivo de contribuir para a literatura sobre sincronização dos ciclos econômicos entre países da América Latina, inicialmente são estimadas medidas de correlações dinâmicas às taxas de crescimento do PIB real usando o modelo dynamic conditional correlation (DCC-GARCH), de Engle (2002). Posteriormente, utilizando as correlações dinâmicas encontradas, estimouse duas regressões em painel para testar os efeitos das crises e os determinantes das correlações entre os países.

Entre os resultados encontrados, destaca-se a importância do comércio bilateral na sincronização dos ciclos dos países latino-americanos, embora as flutuações econômicas desses países estejam muito mais alinhadas aos movimentos da economia norte-americana do que correlacionadas entre si. Esse resultado sugere que os países analisados sofrem mais influências do contexto econômico internacional do que de crises domésticas de países vizinhos.

O presente artigo está estruturado em quatro seções, além desta introdução. Na segunda seção são apresentados trabalhos prévios a respeito de sincronização de ciclos de negócios e análises para a América Latina. Na terceira seção são retratados os dados e os procedimentos metodológicos utilizados. Na quarta seção examina-se os resultados obtidos, e a quinta seção apresenta os principais resultados e possíveis investigações futuras nas considerações finais.

\section{Revisão de literatura}

Mitchell (1927) deu início às análises empíricas dos ciclos econômicos na década de 1920, buscando entender como os choques se propagam ao longo do tempo. No artigo seminal Measuring Business Cycle, Burns e Mitchell (1947) examinaram os ciclos da economia americana e classificaram as variáveis como leading, coincident e lagging, de acordo com o seu comportamento. Segundo os autores, os ciclos de negócios podem ser definidos como um tipo de flutuação 
encontrada na atividade econômica dos países. Um ciclo consistiria de expansões ocorridas ao mesmo tempo em diversas atividades econômicas, seguidas por recessões similares, formando contrações e expansões que seriam recorrentes, mas não periódicas. Em seu trabalho, os autores iniciaram o estudo de tendências a partir de uma média móvel simples. Desde então, foram várias as técnicas desenvolvidas para a determinação dos ciclos. Hamilton (1989) deu seguimento aos estudos para determinar a natureza da tendência de longo prazo, apresentando técnicas para extração de tendência, em que desenvolveu um filtro iterativo não linear baseado em um algoritmo capaz de explicar mudanças de regime na taxa de crescimento.

A literatura que trata da sincronização de ciclos de negócios é ampla e pode ser subdividida em diferentes ramificações. Em uma dessas ramificações, os autores procuram as razões econômicas para a sincronização, como o caso do comércio internacional. No que condiz à teoria econômica, o impacto de uma maior integração comercial na sincronização dos ciclos de negócios é ambígua. $\mathrm{O}$ paradigma de Heckscher-Ohlin prevê que uma maior abertura comercial leva ao aumento da especialização na produção e do comércio interindústria. Se os ciclos de negócios são dominados por choques de indústrias específicas, espera-se que uma maior integração financeira, permitindo o aprofundamento da especialização, leve a um decréscimo das correlações dos ciclos de negócios. Contudo, se os padrões de especialização na produção e o comércio internacional são dominados pelo comércio intraindústria, o aumento do comércio não significará uma maior especialização, e os efeitos na correlação dos ciclos de negócios serão positivos. (Krugman; Obstfeld, 2001).

Imbs (2004) sugere um modelo de comércio internacional considerando as interações entre comércio, finanças, especialização setorial e a sincronização de ciclos de negócios e analisa o efeito de transbordamento de choques entre países. $\mathrm{O}$ autor indica um forte efeito do comércio na sincronização dos ciclos de negócios, porém, com maior magnitude no comércio intraindústria. Frankel e Rose (1998) estimaram o impacto direto do comércio na sincronização dos ciclos de negócios e encontraram uma relação positiva e robusta, que, segundo os autores, é uma indicação de que a especialização induzida pelo comércio tem apenas um pequeno efeito sobre os ciclos de negócios. Baxter e Kouparitsas (2004) analisam determinantes nos comovimentos de ciclos de negócios e confirma a robustez do comércio bilateral, em que países com maior intensidade de comércio possuem 
ciclos mais correlacionados. Monnet e Puy (2016) investigam se a globalização aumentou a sincronização de ciclos de negócios a nível global. Contrariando outros trabalhos, os autores constataram que o ciclo de negócios mundial era tão forte no período Bretton Woods (1950-1971) quanto no período de Globalização (19842006). Por outro lado, apesar de não afetar o nível médio de comovimento, a integração comercial e financeira afeta fortemente a maneira como os países comovimentam com o resto do mundo.

No setor financeiro, a transmissão transfronteiriça de choques financeiros tem tido um papel central na integração financeira de mercados e tem sido uma fonte importante de contágio de crises financeiras. Contudo, não há um consenso quanto ao efeito da integração financeira na sincronização de ciclos de negócios. Kalemli-Ozcan, Soresen e Yosha (2002) analisam o efeito da integração financeira na especialização e, via efeito na especialização, afetaria negativamente a sincronização de ciclos de negócios. Enquanto Imbs (2004) sugere, a partir de um modelo de mínimos quadrados em três estágios, que regiões com fortes ligações financeiras são significativamente mais sincronizadas.

Anatonakis e Sharler (2012) analisam a presença de sincronização de ciclos de negócios entre os países do grupo G7 durante as recessões americanas entre o período de 1960 e 2009. Utilizando as correlações obtidas a partir de um modelo DCC-GARCH e um modelo de dados em painel, os autores concluem que a sincronização entre esses países aumenta em períodos de recessão. Anatonakis (2012) expande essa análise para o período de 1870 a 2010 e conclui que o efeito das recessões depende do período e do nível de globalização analisado. Contudo, verificam que a recessão de 2007-2009 levou a um aumento de sincronização a níveis sem precedentes, possivelmente devido ao aumento significativo da integração financeira nas últimas décadas.

De acordo com Dai (2014), o efeito da integração financeira na sincronia dos ciclos depende da forma como é mensurada ou qual proxy é utilizada. Já Cerqueira e Martins (2009) utilizam a técnica de dados em painel para investigar os determinantes da sincronização de ciclos de negócios de um grupo de países desenvolvidos, e seus resultados mostram um efeito positivo do comércio na sincronização dos ciclos, mas um efeito negativo para a abertura econômica.

Fries e Kappler (2015) investigam os efeitos do Investimento Estrangeiro Direto no comovimento dos ciclos de negócios também por meio do método de 
dados em painel, e seus resultados sugerem que a ligação por meio do investimento estrangeiro direto contribui positivamente para a sincronização dos ciclos, enquanto os efeitos da integração comercial para a similaridade dos ciclos são menos robustos. Já Montinari e Stracca (2016) analisam o efeito transbordamento de ciclos de negócios e sugerem que a integração comercial promove o efeito transbordamento, enquanto a integração financeira apresentou resultados ambíguos: a ligação financeira entre dois países não apresenta efeito significativo, mas a abertura financeira aumenta o efeito transbordamento de ciclos de negócios.

Já outros autores preocuparam-se em encontrar um ciclo econômico comum. Os estudos acerca da sincronização de ciclos de negócios na Europa são amplos e ambíguos. Camacho, Perez-Quirós e Saiz (2006) discutem como o grau de sincronização entre ciclos de negócios de diferentes países podem ser medidos e testados e concluem não existir um ciclo econômico comum no continente europeu. Já Artis, Krolzig e Toro (2004) buscam identificar um ciclo comum europeu através das cadeias de Markov e sugerem que um componente comum governa a dinâmica dos ciclos de negócios europeu. Inklaar, Jong-A-Pin e Haan (2008) concluem que a convergência de políticas monetárias e fiscais tem um impacto significativo na sincronização dos ciclos de negócios. Porém, empiricamente, os resultados novamente são não conclusivos. Baxter e Kouparitsas (2005) argumentam que as uniões monetárias não teriam efeitos robustos no sincronismo dos ciclos de negócios, enquanto Camacho, Perez-Quirós e Saiz (2008) apresentam evidências de que ainda há diferenças entre os ciclos de negócios na Europa. Aguiar-Conraria e Soares (2011) utilizam a análise wavelet para avaliar a sincronização de ciclos de negócios na área do euro e concluem que a proximidade dos ciclos de negócios está altamente correlacionada com a proximidade física. Assim, países mais próximos tendem a ter uma economia mais sincronizada. Bekiros, Nguyen, Uddin e Sjö (2015) partem da análise de wavelet para detectar e identificar a sincronização e convergência de ciclos de negócios na Área do Euro antes e depois da crise financeira. Os resultados encontrados confirmam que a sincronização aumenta na Área do Euro, em que o comovimento parece ser acumulado e se intensificou nas bandas de frequência de médio prazo, principalmente durante a crise financeira. Nesse cenário, as políticas econômicas adotadas pelo Banco Central Europeu, devido ao aumento da sincronização durante o período da crise, foram instrumentos de estabilização efetivos. Contudo, observadas mudanças nas relações lead-lag das economias europeias, a 
convergência e a sincronização variam fortemente em diferentes bandas de frequência e horizontes temporais.

Outros autores buscaram entender a sincronização de ciclos econômicos em economias emergentes. Comin, Loayza, Pasha e Serven (2014) analisam os ciclos de negócios para países em desenvolvimento a partir de um modelo DSGE para dois países, e concluem que choques originados em países em desenvolvimento possuem efeitos maiores e mais persistentes em países em desenvolvimento. Calderón, Chong e Stein (2007) analisam se há diferenças na relação entre a integração financeira e os comovimentos dos ciclos de negócios quando se trata de países desenvolvidos e países em desenvolvimento. Seus resultados sugerem que a intensidade de comércio entre países industrializados tem efeito positivo na correlação dos ciclos do produto, enquanto entre países em desenvolvimento, esses efeitos são menores. Dai (2014) examina a sincronização de ciclos de negócios na Ásia, utilizando Estados Unidos, Japão e China como países de referência para as flutuações econômicas da região. Através da análise de dados em painel, encontram evidências empíricas do impacto da integração financeira, comercial e de similaridade de políticas econômicas como determinantes da sincronização dos ciclos de negócios.

Clark e van Wincoop (2001), Perez-Quiros e Saiz (2006) e Furceri (2004) investigam os efeitos da coordenação de política fiscal na sincronização de ciclos na União Europeia e encontram uma relação positiva entre coordenação de política fiscal e condições de déficits similares e sincronização de ciclos. Para a política monetária, entretanto, não há o mesmo consenso. Arthis e Zang (1997) concluem que em caso de pouca sincronização entre os países, políticas monetárias comuns tendem a aumentar a divergência entre os ciclos, enquanto para países mais sincronizados, a política comum leva a uma convergência dos ciclos reais.

Antonakakis e Tondl (2014) também pesquisam o efeito da coordenação da política fiscal e monetária na sincronização de ciclos na União Europeia. Usando um modelo de equações simultâneas, os autores constatam que a falta de disciplina fiscal de alguns países da União Europeia impede maior sincronização de ciclos entre os países. À medida que a diferença do déficit orçamentário desses países diminui, aumenta-se a convergência dos ciclos. Quanto à política monetária, os autores concluem que a sincronização de ciclos aumentou após a adoção do euro, 
implicando que quanto maior a coordenação de política monetária entre os países, mais sincronizados ficam ao longo do tempo.

Para a América Latina, Engel e Issler (1993) analisaram comovimentos de curto e longo prazo para Brasil, Argentina e México e concluíram que os três países seguem uma mesma tendência de crescimento e de flutuações de curto prazo. Já Mejía-Reyes (2000) sugere que não existe um ciclo comum entre Brasil, Argentina, Colômbia, Chile, México, Peru, Venezuela e Estados Unidos. Arnaudo e Jacobo (1997) concluem que os comovimentos para os países do Mercosul são pouco uniformes, apresentando relação de ciclos apenas para Brasil e Argentina. Gonçalves (2003) investigou a relação entre o comércio bilateral e os ciclos econômicos para a América Latina e sugere que países que comercializam mais entre si apresentam ciclos mais sincronizados.

Adler e Sosa (2012) examinam a influência do Brasil nos outros países da América do Sul a partir de um modelo VAR. Seus resultados apontam que devido à integração comercial, o Brasil é uma importante fonte de choques para os países do Cone Sul (Argentina, Bolívia, Chile, Paraguai e Uruguai). Contudo, para as economias vizinhas menores, os efeitos não são significativos. Vélez e Giraldo (2015) testam a sincronia dos ciclos na América Latina para o período entre 1980 e 2014 a partir do algoritmo de Bry-Boschan aplicado para o produto interno bruto e para o índice de produção industrial desses países e medem o grau de sincronização a partir do indicador de Harding e Pagan e não encontram uma grande sincronização entre esses países, com exceções para Chile-México e Chile-Colômbia.

\section{Dados e metodologia}

Para estimar as correlações entre os países da América Latina, utilizou-se a taxa de crescimento do PIB real para do período entre 1968 e 2016, obtidas a partir da diferença logarítmica das séries do PIB reais. As séries anuais foram interpoladas para obtê-las em frequência trimestral. Tal procedimento foi necessário devido à indisponibilidade de séries trimestrais para os países analisados que sejam suficientemente longas para a estimação dos modelos GARCH, que perdem precisão com um número reduzido de observações. Escolheu-se Argentina, Brasil, Chile e México para análise, tanto pelo fato de representarem as maiores economias da América Latina quanto pela disponibilidade de dados dos países menores. 
As dummies utilizadas para verificar os efeitos das crises internacionais foram criadas a partir dos períodos de recessão definidos pelo National Bureau for Economic Research Business Cycle Dating Committee, enquanto as crises domésticas foram criadas baseadas nos períodos recessivos de cada país.

Para analisar se uma coordenação de política monetária aumenta ou diminui a sincronização de ciclos, empregou-se como variável o diferencial das taxas de juros utilizadas pelos Bancos Centrais como instrumento de política monetária, disponibilizada pelo Banco de Compensações Internacionais (BIS). Já para coordenação de política fiscal, utilizou-se o diferencial dos gastos do governo como percentual do PIB, disponível no Government Finance Statistics (GFS), base de dados do Fundo Monetário Internacional (FMI).

Diversas são as medidas propostas na literatura para medir relações de comércio bilaterais e de intensidade financeira. A utilizada neste trabalho é a construída por Frankel e Rose (1998) e utilizada por Fidrmuc (2005), Siedchlag (2010) e Antonakakis e Tondl (2014).

De acordo com Antonakakis e Tondl (2014), os indicadores de Frankel e Rose (1998) são mais adequados do que indicadores como o proposto por Imbs (2004) e Fidrmuc, Iwatsubo e Ikeda (2012), uma vez que essas medidas possuem a soma do PIB no denominador. Isso estabelece uma relação com o tamanho das economias, o que não é necessariamente válido quando a medida do comércio bilateral estiver relacionada ao volume total do comércio.

Deste modo, a influência da intensidade do comércio é medida pela variável de comércio bilateral, construída a partir da sugestão de Frankel e Rose (1998):

$$
T_{i, j, t}=\frac{\left(X_{i, j, t}+M_{i, j, t}\right)}{\left(X_{i, t}+X_{j, t}+M_{i, t}+M_{j, t}\right)}
$$

em que $X_{i, j, t}$ são as exportações do país $i$ para o país $j$ no período $t,{ }^{M_{i, j, t}}$ são as importações do país $i$ para o país $j$ no período $t$.

Para a intensidade financeira entre os países, utilizou-se o fluxo de investimento bilateral total, construído de maneira similar à variável de comércio bilateral:

$$
B I_{i, j, t}=\frac{\left(I_{i j, t}+I_{j i, t}\right)}{\left(I_{i, t}+I_{j, t}\right)}
$$


em que $I_{i j, t}$ é a variável de investimento no país $i$ no país $j$ no período $t$.

As variáveis de intensidade de comércio e fluxo de investimento foram construídas a partir dos dados das bases Direction on Trade Statistics (DOTS) e da International Financial Statistics (IFS), ambas também disponibilizadas pelo FMI.

Antes dos procedimentos econométricos, utilizou-se os testes de DickeyFuller Aumentado (ADF) e Phillips-Perron, que rejeitaram a hipótese de raiz unitária para todas as análises, assegurando, assim, a estacionariedade das séries utilizadas neste trabalho.

\subsection{DCC-GARCH}

O primeiro passo da análise consiste na estimação das correlações condicionais entre as taxas de crescimento dos países. Seguindo Antonakakis e Sharler (2012), Antonakakis (2012), utilizou-se dos modelos Multivariate Generalized Autoregressive Conditional Heteroskedacity (MGARCH) para a análise das correlações. Os modelos MGARCH são uma extensão dos modelos GARCH univariados que permitem predizer a dependência entre comovimentos entre séries de retornos de ativos, uma vez que as volatilidades financeiras podem mover-se juntas mais ou menos próximas ao longo do tempo e dos mercados (Orskaug, 2009). Desta forma, os modelos GARCH multivariados têm sido usados para investigar transmissão efeitos spillover e contágio entre mercados.

A estimação dos modelos DCC-GARCH ocorre em dois estágios: no primeiro, cada variância condicional é especificada como um processo GARCH univariado, e no segundo, os resíduos padronizados da primeira etapa são utilizados para construir a matriz de correlação condicional. Portanto, o modelo DCC pode ser especificado da seguinte maneira:

$$
\begin{gathered}
y_{t}=\mu_{t}+\varepsilon_{t} \\
\varepsilon_{t} \mid \Omega_{-1} \sim N\left(0, H_{t}\right)
\end{gathered}
$$

em que os resíduos do processo são modelados como:

$$
\varepsilon_{t}=\mathbf{H}_{t}^{\frac{1}{2}} \eta_{t}
$$

em que ${ }^{\varepsilon_{t}}$ é um vetor $N \times 1$ da média corrigida dos retornos de $N$ no tempo $t$, isto é, $\mathrm{E}\left[\varepsilon_{t}\right]=0$ e $\operatorname{cov}\left[\varepsilon_{t}\right]=\mathbf{H}_{t} . \mu_{t}$ é o vetor da média condicional de ${ }^{y_{t}}$, e pode ser 
modelado como um VAR ou apenas representar a média não condicional dos retornos. ${ }^{\varepsilon_{t}}$ é o vetor de resíduos baseados no conjunto de informações $\Omega$, disponível no período $t$-1. $\mathbf{H}_{t}$ é uma matriz das covariâncias condicionais de $\varepsilon_{t}$ no tempo $t$ e é positiva definida. $\mathbf{H}_{t}^{\frac{1}{2}}$ é uma matriz $N \times N$ no tempo $t$ tal que $\mathbf{H}_{t}$ é a matriz de variância condicional de ${ }^{a_{t}} . \mathbf{H}_{t}^{\frac{1}{2}}$ pode ser obtida por uma fatorização de Cholesky de $H_{t}$. E $z_{t}$ é um vetor $N \times 1$ de erros i.i.d. tal que $\mathrm{E}\left[\eta_{t}\right]=0$ e $\mathrm{E}\left[\eta_{t} \eta_{t}^{T}\right]=I$. O modelo de correlação dinâmica surgiu como uma extensão de Engle (2002) e Tse e Tsui (2002) ao modelo CCC-GARCH de Bollerslev (1990). No DCC-GARCH, a matriz de covariância condicional é definida por:

$$
\mathbf{H}_{t}=\mathbf{D}_{t} \mathbf{R}_{t} \mathbf{D}_{t}
$$

onde $\mathbf{D}_{t}=\operatorname{diag}\left(h_{t}^{1 / 2}, \ldots, h_{N t}^{1 / 2}\right)$ e $\mathbf{R}$ é a matriz de correlação condicional. A dinâmica de $R_{t}$ é dada por:

$$
R_{t}=\operatorname{diag}\left\{Q_{t}\right\}^{-1} Q_{t} \operatorname{diag}\left\{Q_{t}\right\}^{-1}
$$

$\mathrm{Ou}$

$$
\rho_{i, j, t}=\frac{q_{i, j, t}}{\sqrt{q_{i, i, t} q_{j, j, t}}}
$$

com os elementos a diagonal principal são iguais a 1 e os outros elementos iguais às correlações condicionais dinâmicas em que $\eta_{t}=\left(\eta_{1, t}, \ldots, \eta_{N, t}\right)^{\prime}$ é o vetor de resíduos padronizados. $\bar{Q}_{t}$ é a matriz de covariância não condicional de $\eta_{t}$ e $a$ e $b$ são escalares não negativos que devem satisfazer $a+b<1$; $Q_{0}$ é positiva definida. O modelo DCC é estimado por quasi-máxima verossimilhança (QML) considerando uma distribuição $t$ de student multivariada, visto que a suposição de normalidade dos resíduos é rejeitada.

\subsection{Dados em painel}

O segundo passo da análise consiste na estimação de dois painéis: o primeiro para a verificação da sincronização dos países nos períodos de crises e o segundo para tentar encontrar os determinantes dessa sincronização. 
A ideia no modelo de efeitos fixos é controlar os efeitos das variáveis que foram omitidas do modelo e que podem ser diferentes entre os diferentes países, mas constantes no tempo. Para capturar a diferença entre as diferentes unidades de corte transversal, toma-se o coeficiente de intercepto como constante ao longo do tempo e variando entre as observações. No caso dos coeficientes angulares, estes são considerados constantes entre as unidades micro e ao longo do tempo (FRIES e KAPPLER, 2015).

Assim, de acordo com Wooldridge (2010), as suposições a serem feitas sobre o modelo são as seguintes:

$$
\beta_{1 i t}=\beta_{1} ; \beta_{2 i t}=\beta_{2} ; \beta_{1 i t}=\beta_{3} ; \beta_{k i t}=\beta_{k} .
$$

O modelo de efeitos fixos é representado, então, por:

$$
Y_{i t}=\beta_{1 i}+\beta_{2} X_{2 i t}+\beta_{3 i t}+\ldots+\beta_{k} X_{k i t}+\mu_{i t}
$$

que em sua forma matricial fica escrito da seguinte maneira:

$$
\left[\begin{array}{c}
Y_{i 1} \\
Y_{i 2} \\
\vdots \\
Y_{i T}
\end{array}\right]=\left[\begin{array}{ccccc}
1 & X_{1 i 1} & X_{2 i 1} & \cdots & X_{k i 1} \\
1 & X_{1 i 2} & X_{2 i 2} & \cdots & X_{k i 2} \\
\vdots & \vdots & \vdots & \ddots & \vdots \\
1 & X_{1 i T} & X_{2 i T} & \cdots & X_{k i T}
\end{array}\right]\left[\begin{array}{c}
\beta_{i 1} \\
\beta_{i 2} \\
\vdots \\
\beta_{i k}
\end{array}\right]+\left[\begin{array}{c}
\mu_{i 1} \\
\mu_{i 2} \\
\vdots \\
\mu_{i T}
\end{array}\right]
$$

em que ${ }^{\beta_{i 1}}$ refere-se aos coeficientes do intercepto a serem estimados para cada correlação entre os países $i j$.

Segundo Wooldridge (2010), para diferenciar o intercepto de uma observação para a outra, faz-se a utilização de variáveis dummies (variáveis binárias) no modelo a ser estimado. Stock e Watson (2003) salientam que as variáveis binárias refletem todas as variáveis omitidas que diferem de uma observação para outra, mas que são constantes ao longo do tempo.

O modelo com variáveis binárias pode ser representando como:

$$
Y_{i t}=\beta_{1,1}+\beta_{2} X_{2 i t}+\beta_{3} X_{3 i t}+\ldots+\beta_{k} X_{k i t}+\beta_{1,2} D_{2 i}+\beta_{1,3} D_{3 i}+\ldots+\beta_{1, n} D_{n, i}+\mu_{i t}
$$

em que $D_{n i}$ expressa a variável binária para cada unidade micro, sendo igual a um quando $1=n$ e zero contrário. Nota-se que são introduzidas $n-1_{\text {variáveis }}$ 
binárias, a fim de evitar o problema da multicolinearidade. Neste caso, para $i=1_{\mathrm{O}}$ intercepto é dado por $\beta_{1,1}$. Para $i \geq 2$ o intercepto é representado por $\beta_{1,1}+\beta_{1, i}$.

\section{Resultados}

A estimação dos modelos DCC-GARCH inicia-se pelos modelos GARCH univariados. Como testado por Engle e Sheppard (2001) e pontuado por Capello, Engle e Shepard (2006), a estimação dos modelos univariados possui poucos efeitos no modelo final, uma vez que esses modelos produzem padrões de volatilidade relativamente similares e não afetam os sinais dos resíduos padronizados. Não sendo necessário, assim, testar uma infinidade de modelos, uma vez que as correlações condicionais são relativamente insensíveis aos modelos univariados dentro de uma determinada classe.

A especificação dos modelos foi selecionada utilizando o Critério de Informação Bayesiano (BIC) e log-likelihood (LL) a partir de uma série de modelos com diferentes especificações de lags. Assim, o modelo escolhido foi o que apresentou menor BIC. Utilizou-se o DCC de ordem comum $(1,1)$ e testou-se a mesma ordem para o caso univariado para todas as variáveis com as classes GARCH e EGARCH, assim como de ordem $(2,2)$ e distribuição normal.

\section{Tabela 1 - Modelos DCC-GARCH e critérios de seleção}

\begin{tabular}{cccc}
\hline Modelo & \# de Parâmetros & BIC & Log-like \\
\hline \hline DCC-GARCH $(1,1)$ & 36 & 6,921 & $-579,965$ \\
DCC-EGARCH $(1,1)$ & 44 & 7,025 & $-568,998$ \\
DCC-GARCH $(2,2)$ & 52 & 6,771 & $-52,993$ \\
DCC-EGARCH $(2,2)$ & 56 & 6,590 & $-494,890$ \\
\hline
\end{tabular}

Fonte: Resultados da pesquisa.

A Tabela 1 mostra os valores do BIC e LL para os melhores modelos, que sugere como melhor especificação o modelo $\operatorname{DCC}-\operatorname{EGARCH}(2,2)$, sendo este o modelo escolhido para a análise presente. A média condicional ${ }^{\mu}$ da equação (7) foi modelada seguindo um VAR (5), cuja ordem foi escolhida pelos critérios de Akaike (AIC), Hannah-Quinn (HQ) e Schwarz (SC). 
Tabela 2 - Resultados do Modelo DCC

\begin{tabular}{lccc}
\hline \multicolumn{1}{c}{$\rho$} & Argentina & Brasil & Chile \\
\hline \hline Brasil & $\begin{array}{c}0,210 * * * \\
(-1,26 \mathrm{E}-08)\end{array}$ & & \\
& & \\
\hline Chile & $0,383 * * *$ & $0,309 * * *$ & \\
& $(-2,20 \mathrm{E}-16)$ & $(-2,20 \mathrm{E}-16)$ & \\
\hline México & 0,021 & $0,213 * * *$ & $0,206 * * *$ \\
& $(-0,539)$ & $(-9,56 \mathrm{E}-08)$ & $(-2,1 \mathrm{E}-08)$ \\
\hline$\alpha$ & 0,489 & & \\
& 0,000 & & \\
\hline$\beta$ & 0,374 & & \\
& 0,000 & & \\
\hline
\end{tabular}

Fonte: Resultados da pesquisa.

A Tabela 2 apresenta os resultados do modelo DCC. De acordo com a tabela, das 6 correlações condicionais dinâmicas, 5 são significativas, e aparentemente, são diretamente influenciadas pela proximidade geográfica. A Figura 1 mostra as séries correlações condicionais dinâmicas para os países da América Latina. A linha tracejada azul mostra a média das correlações, enquanto as linhas vermelhas assinalam um desvio padrão acima e abaixo da média. Chama a atenção a alta volatilidade dessas correlações, variando entre valores positivos e negativos, embora apenas em períodos específicos esses valores ultrapassem um desvio padrão acima ou abaixo. Esses resultados refletem a instabilidade econômica dessas economias, que alternam períodos de grande crescimento com quedas repentinas em suas variáveis econômicas. Diferente, por exemplo, do resultado encontrado por Antonakakis e Scharler (2012) para o G7, cujas correlações, apesar de apresentarem alguns picos positivos e negativos, no geral, apresentam menor variabilidade ao longo do tempo. 


\section{Figura 1 - Correlações Condicionais Dinâmicas (1968-2016)}
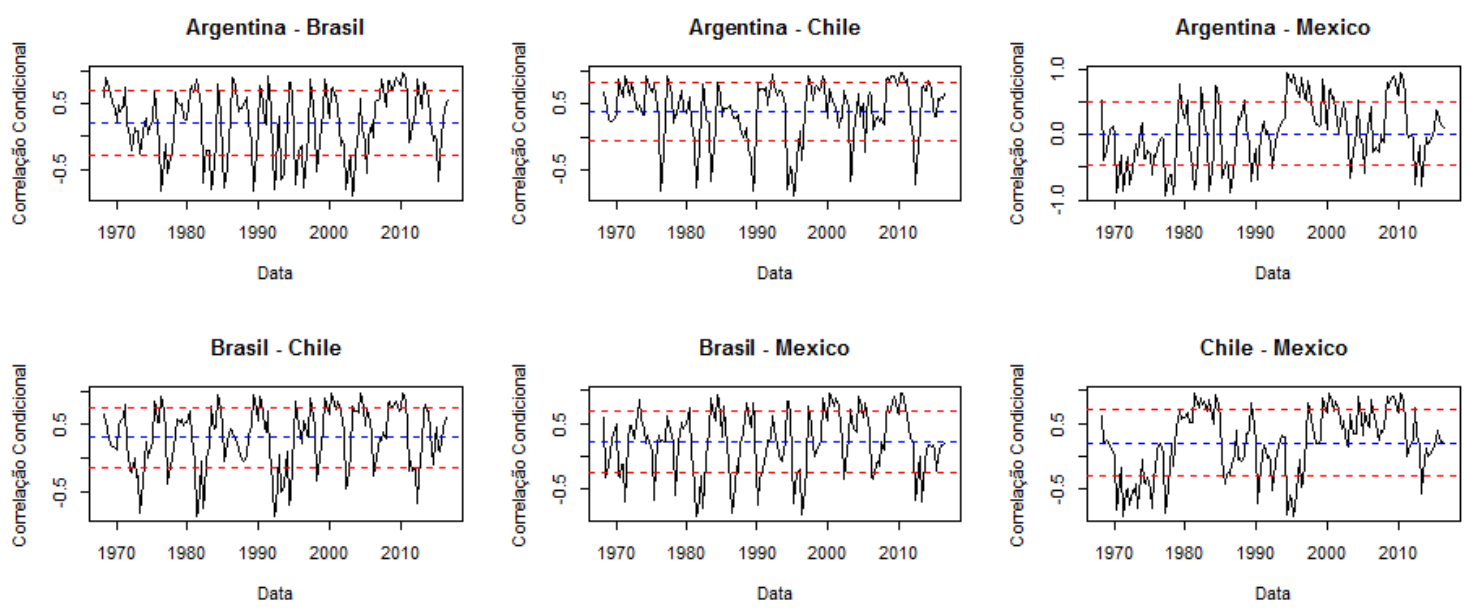

Fonte: Resultados da pesquisa.

A figura 2 mostra a janela de correlações condicionais a partir de 1990, período que marcou a abertura econômica e financeira nos países latinoamericanos. Neste gráfico, é possível perceber que os períodos de quedas ou aumentos bruscos de correlação, que ultrapassam um desvio padrão com relação à média, coincidem com os períodos de crises domésticas ou de maior instabilidade local e crises internacionais. Destacam-se nesse período, as crises do México (1994), da Argentina (2001), e do Brasil (2014), além das crises internacionais nesse período, uma pequena recessão em 2001 e a crise financeira em 2008, que, como mostram os gráficos, são os períodos de maiores picos nas correlações: crises domésticas aumentam a correlação negativa, ou seja, o país em crise apresenta crescimento negativo, enquanto os outros países seguem com o crescimento positivo, não sendo diretamente afetados pela crise vizinha. $\mathrm{O}$ país que aparenta maior instabilidade é a Argentina, que além dos períodos de crise, passou por um período instável em 2012, saindo de um crescimento de 61\% em 2011 para -11\% em 2012, o que refletiu como um pico negativo na correlação com os outros países, uma vez que esses não foram atingidos. Uma exceção é para a crise da Argentina, que no período que ocorreu, em 2001, as correlações entre os países foram positivas, cabendo lembrar que coincidiu com uma pequena recessão americana em 2001, que sempre possui um impacto maior no México. Assim, tanto na recessão de 2001 quanto na crise financeira de 2008, houve uma maior sincronização entre esses países, sugerindo que essas economias tendem a "caminhar juntas" apenas em períodos de maior influência internacional. 


\section{Figura 2 - Correlações Condicionais Dinâmicas (1990-2016)}
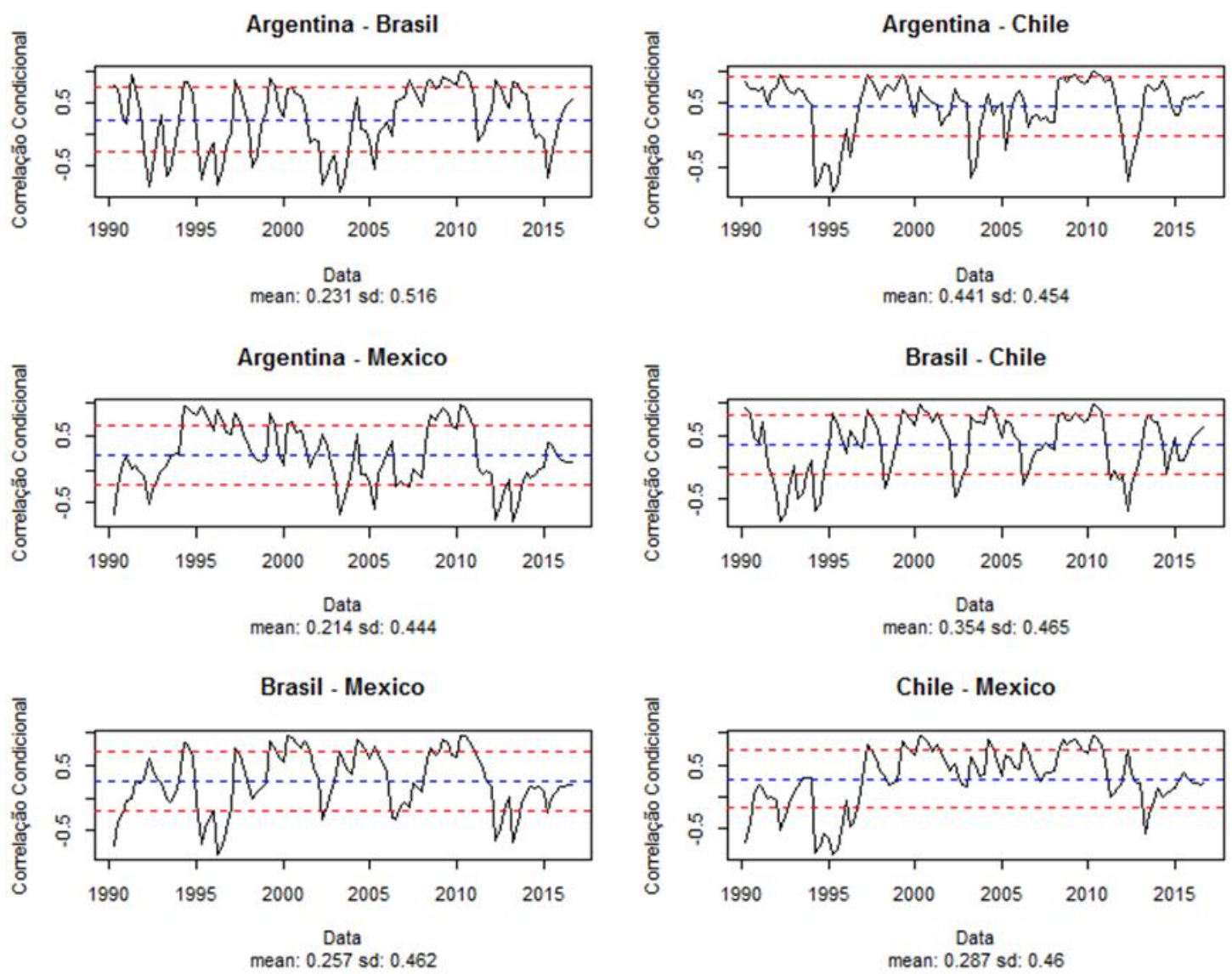

Fonte: Resultados da pesquisa.

Para identificar mais indícios dessa relação entre os países latinoamericanos e suas crises, testa-se formalmente a hipótese de que os países sincronizam (ou dessincronizam) durante crises no período entre 1990 e 2016. Para isso, utilizou-se a transformada $Z$ de Fisher para normalizar as correlações condicionais dinâmicas, $\rho_{i, j, t}$ e estimou-se o seguinte painel de efeitos fixos:

$$
z \rho_{i j, t}=\alpha_{i j}+\beta_{1} \text { trend }+\beta_{2} \text { rec }_{t}+\beta_{3} \arg _{t}+\beta_{4} \text { bra }_{t}+\beta_{5} m e x_{t}+\varepsilon_{i, j, t}
$$

em que ${ }^{z \rho_{i j, t}}$ é a transformada $Z$ de Fisher de $\rho_{i j, t}, \alpha_{i j}$ são os efeitos fixos crosssection, trend é um termo de tendência linear, ${ }^{r e c}{ }_{t}$ denota uma variável dummy que é igual a 1 se os Estados Unidos estão em recessão no período $t$ e igual a 0 caso contrário; ${ }^{a r g}{ }_{t},{ }^{b r a_{t}} \mathrm{e}^{m e x_{t}}$ são variáveis dummy que possuem valor 1 para as crises domésticas da Argentina, Brasil e México, respectivamente e 0 caso contrário. 


\section{Tabela 3 - Sincronização de ciclos de negócios durante crises e recessões (1990-2016)}

\begin{tabular}{cccc}
\hline & $\beta$ & Erro Padrão & $\begin{array}{c}\mathrm{P}- \\
\text { valor }\end{array}$ \\
\hline \hline trend & 0,002 & 0,000 & 0,000 \\
rec & 0,134 & 0,053 & 0,013 \\
arg & 0,177 & 0,055 & 0,001 \\
bra & $-0,19$ & 0,068 & 0,007 \\
mex & $-0,26$ & 0,067 & 0,000 \\
\hline $\mathrm{n}=648$ & & $\mathrm{R} 2=0.1$ & \\
\hline
\end{tabular}

Fonte: Resultados da pesquisa.

Os resultados são apresentados na tabela 3. É possível perceber que os resultados corroboram o que era perceptível nas séries de correlação: os países latino-americanos sincronizam durante crises internacionais e dessincronizam nos períodos de crises domésticas. Ou seja, apesar de correlacionados, esses países não possuem influência, ou não são integrados o suficiente para que uma crise doméstica atinja os países vizinhos. A exceção para os resultados fica para o resultado da Argentina, que possui um sinal positivo, sugerindo sincronização. Porém, como já citado, a crise argentina coincidiu com uma recessão americana em 2001, que pode ter influenciado para que os países acompanhassem o momento ruim da economia. E como esperado, as crises americanas possuem impacto significativo em todos os países, colaborando para a sincronia desses nesses períodos de crise.

Em seguida, analisou-se quais seriam os determinantes da sincronia entre os países da América Latina. Para isso, estimou-se um painel de efeitos fixos para verificar se a coordenação de política monetária e política fiscal, além de comércio e fluxo de investimento bilaterais teriam efeitos sobre as correlações dinâmicas entre os países. Neste caso, a análise foi feita para o período entre 2001 e 2016, devido à indisponibilidade de dados para os anos anteriores. A regressão em painel seguiu a seguinte forma:

$$
z \rho_{i j, t}=\alpha_{i j}+\beta_{1} p f_{i j, t}+\beta_{2} p m_{i j, t}+\beta_{3} b t_{i j, t}+\beta_{4} b i_{i j, t}+\varepsilon_{i, j, t}
$$


em que ${ }^{z \rho_{i j, t}}$ é a transformada Z de Fisher de $\rho_{i j, t},{ }^{\alpha_{i j}}$ são os efeitos fixos crosssection, $p f_{i j, t}$ é o diferencial de gastos do governo, e representa a diferença de política fiscal entre os países $i j$ no período $t, p m_{i j, t}$ é o diferencial de taxa de juros, que representa a diferença entre a política monetária dos países ij no período $t,{ }^{b t_{i j, t}}$ denotam o comércio e o fluxo de investimento nos países $i j$ no período $t$ respectivamente.

\section{Tabela 4 - Determinantes da sincronização de ciclos de negócios na América Latina}

\begin{tabular}{lrrr}
\hline & \multicolumn{1}{c}{$\beta$} & Erro-Padrão & P-valor \\
\hline \hline pf & 0,238 & 0,058 & 0,000 \\
pm & 0,021 & 0,029 & 0,467 \\
bt & 0,234 & 0,089 & 0,009 \\
bi & 0,033 & 0,030 & 0,272 \\
\hline $\mathrm{n}=324$ & \multicolumn{3}{c}{ R2 $=0,650$} \\
\hline
\end{tabular}

Fonte: Resultados da pesquisa.

A tabela 4 apresenta os resultados para essa estimação. Os resultados sugerem que quanto maior o diferencial de gastos do governo, ou seja, quanto menos coordenada a política fiscal, maior a sincronização de ciclos. Ainda não há um consenso na literatura sobre qual deve ser o efeito da política fiscal na sincronização de ciclos. Países que possuem maior convergência fiscal tendem a ser mais sincronizados, enquanto países marcados pela irresponsabilidade fiscal estão menos propensos à sincronização, uma vez que são mais suscetíveis a choques fiscais. Por outro lado, os países estão suscetíveis a choques assimétricos e mesmo choques similares podem ter efeitos diversos nesses países por possuírem diferentes mecanismos de propagação. Se estabilizadores fiscais automáticos ou uma política fiscal discricionária é utilizada para compensar essas assimetrias, um maior diferencial de política fiscal pode gerar ciclos econômicos mais sincronizados (Darvas et al.; 2005; Kose et al., 2003)

O diferencial de política monetária também apresenta uma relação positiva, porém não significativa. Entretanto, como pontua Dai (2014), não está claro como o diferencial de taxa de juros de curto prazo pode afetar a correlação entre duas economias. Por um lado, se duas economias são similares e possuem políticas 
monetárias similares, espera-se que após um choque, reajam de maneira similar. Nesse caso, espera-se que quanto mais coordenadas, ou menor o diferencial de taxa de juros, mais sincronizadas serão essas economias. Por outro lado, se as duas economias possuem regimes de políticas monetárias diferentes, é esperado que reajam de maneiras diferentes a um choque. Deste modo, diferentes políticas monetárias podem levar a um mesmo resultado em ambas as economias, levando assim a aumentar a sincronização dos ciclos, embora os países possuam diferentes políticas monetárias.

Já o comércio bilateral tem uma relação positiva e significativa com a correlação dos países, sugerindo que quanto maior o comércio entre dois países, mais sincronizadas são suas economias. Era esperado que o comércio fosse o principal responsável pela sincronização dos ciclos, uma vez que esses países participam de acordos de livre comércio, como Mercosul e ALADI. Já o fluxo de investimento bilateral possui uma relação positiva, mas não significativa, com as correlações, sugerindo que o fluxo financeiro entre esses países não impacta a sincronização de ciclos dos mesmos. Esse resultado pode ser reflexo dos mercados financeiros ainda pouco desenvolvidos e pouco integrados entre os países analisados.

\section{Considerações finais}

Este trabalho buscou investigar a sincronização de ciclos de negócios das quatro principais economias da América Latina: Argentina, Brasil, Chile e México. O foco da pesquisa foi analisar o padrão da correlação dinâmica entre as taxas de crescimento dos países, como as crises domésticas e internacionais influenciam nesse padrão e quais os possíveis determinantes da sincronização entre os países latinos.

Para a análise, estimou-se as correlações condicionais dinâmicas a partir de um modelo DCC-GARCH e, em seguida estimou-se duas regressões em painel, uma para efeitos da crise e outra para determinantes da sincronização. Os resultados mostram que os países latino-americanos tendem a seguir o mesmo movimento de ciclos em períodos de crises internacionais e a divergir em períodos de crises domésticas. Esses resultados sugerem que os países analisados tendem a ser influenciados muito mais pelo contexto econômico internacional, especialmente americano, do que pelos países vizinhos, o que pode indicar que 
apesar de participarem de acordos de livre comércio, esses países não são integrados o suficiente para que uma crise doméstica em um desses países seja transmitida para os outros.

$\mathrm{Na}$ análise dos determinantes da sincronização, os resultados indicaram que o comércio bilateral é o principal responsável pela sincronização dos ciclos entre países. Já o fluxo de investimento bilateral, embora tenha um efeito positivo, não foi significativo, sugerindo que a integração financeira desses países não influencia a sincronização dos ciclos. As variáveis de coordenação de políticas econômicas mostraram que um maior diferencial de política fiscal possui efeito positivo na sincronização dos ciclos, o que pode ser resultado do uso contracíclico deste tipo de política.

\section{Referências}

ADLER, G.; SOSA, S. Intraregional spillovers in South America: is Brazil systemic after all? The World Economy, v. 37, n. 3, p. 456-480, 2014.

AGUIAR-CONRARIA, L.; AZEVEDO, N.; SOARES, M. Using wavelets to decompose the time-frequency effects of monetary policy. Physica A: Statistical mechanics and its Applications, v. 12, n. 1, p. 2863-2878, 2008.

ANTONAKAKIS, N. Exchange return co-movements and volatility spillovers before and after the introduction of euro. Journal of International Financial Markets, Institutions and Money, v. 22, n. 5, p. 1091-1109, 2012.

ANTONAKAKIS, N.; SCHARLER, J. The synchronization of GDP growth in the G7 during US recessions. Applied Economics Letters, v. 19, n. 1, p. 7-11, 2012.

ANTONAKAKIS, N.; TONDL, G. Does integration and economic policy coordination promote business cycle synchronization in the EU? Empirica, v. 41, n. 3, p. 541-575, 2014.

ARTIS, M.; KROLZIG, H.; TORO, J. The European business cycle. Oxford Economic Papers, v. 56, n. 1, p. 1-44, 2004.

BAXTER, M.; KOUPARITSAS, M. Determinants of business cycle comovement: a robust analysis. Journal of Monetary Economics, v. 52, n. 1, p. 113-157, 2005.

BEKIROS, S.; NGUYEN, D.; UDDIN, G.; SJÖ, B. Business cycle (de)synchronization in the aftermath of the global financial crisis: implications for 
the Euro area. Studies in Nonlinear Dynamics \& Econometrics, v. 19, n. 5, p. 609$624,2015$.

BOLLERSLEV, T. Modelling the coherence in short-run nominal exchange rates: a multivariate generalized ARCH model. Review of Economics and Statistics, v. 72, n. 3, p. 498-505, 1990.

BURNS, A.; MITCHELL, W. Measuring business cycles. New York: National Bureau of Economic Research, 1947.

CALDERON, C.; CHONG, A.; STEIN, E. Trade intensity and business cycle synchronization: are developing countries any different? Journal of international Economics, v. 71, n. 1, p. 2-21, 2007.

CAMACHO, M.; PEREZ-QUIRÓS, G.; SAIZ, L. Are European business cycles close enough to be just one? Journal of Economics Dynamics and Control, v. 30, n. 9, p. 1687-1706, 2006.

CAMACHO, M.; PEREZ-QUIRÓS, G.; SAIZ, L. Do European business cycles look like one? Journal of Economics Dynamics and Control, v. 32, n. 7, p. 21652190, 2008.

CAPPIELLO, L.; ENGLE, R.; SHEPPARD, K. Asymmetric dynamics in the correlations of global equity and bond returns. Journal of Financial Econometrics, v. 4, n. 4, p. 537-572, 2006.

CERQUEIRA, P.; MARTINS, R. Measuring the determinants of business cycle synchronization using a panel approach. Economics Letters, v. 102, n. 2, p. 106$108,2009$.

COMIN, D.; LOAYZA, N.; PASHA, F.; SERVEN, L. Medium term business cycles in developing countries. American Economic Journal: Macroeconomics, $\mathrm{v}$. 6, n. 4, p. 209-245, 2014.

DAI, Y. Business cycle synchronization in Asia: the role of financial and trade linkages. Asian Development Bank Working Paper, n. 139, 2014.

DARVAS, Z.; ROSE, A.; SZAPÁRY, G. Fiscal divergence and business cycle synchronization: irresponsibility is idiosyncratic. National Bureau of Economic Research, 2005.

ENGLE, R. Dynamic conditional correlation: a simple class of multivariate generalized autoregressive conditional heteroskedasticity models. Journal of Business \& Economic Statistics, v. 20, n. 3, p. 339-350, 2002. 
ENGLE, R.; SHEPPARD, K. Evaluating the specification of covariance models for large portfolios. New York University Working Paper, 2008.

FIDRMUC, J. The endogeneity of the optimum currency area criteria, intraindustry trade, and EMU enlargement. Contemporary economic policy, v. 22, n. 1, p. 1-12, 2004.

FIDRMUC, J.; IWATSUBO, K.; IKEDA, T. International transmission of business cycles: evidence from dynamic correlations. Economics Letters, v. 114, n. 3, p. 252-255, 2012.

FRANKEL, J., ROSE, A. The endogeneity of the optimum currency area criteria. Economic Journal, v. 108, p. 1009-1025, 1998.

FRIES, C.; KAPPLER, M. Does foreign direct investment synchronize business cycles? Results from a panel approach. ZEW - Centre for European Economic Research Discussion Paper. n. 15-031, 2015.

HILL, R.; GRIFFITHS, W.; JUDGE, G. Econometria. São Paulo: Saraiva, 2010.

IMBS, J. Trade, finance, specialization, and synchronization. Review of Economics and Statistics, v. 86, n. 3, p. 723-734, 2004.

INKLAAR, R.; JONG-A-PIN, R.; HAAN, J. Trade and business synchronization in OECD countries: a re-examination. European Economic Review, v. 52, n. 4, p. 646-666, 2008.

KALEMLI-OZCAN, S.; SORENSEN, B.; YOSHA, O. Economic integration, industrial specialization, and the asymmetry of macroeconomic fluctuations. Journal of International Economics, v. 55, n. 1, p. 107-137, 2003.

KOSE, A.; PRASAD, E.; TERRONES, M. How does globalization affect the synchronization of business cycles? American Economic Review, n. 93, v. 2, p. 5762, 2003.

KRUGMAN, P.; OBSTFELD, M. Economia internacional: teoria e política. São Paulo: Makron Books, 2001.

MITCHELL, W. Business cycles: the problem and its setting. Nova York: NBER, 1927.

MONNET, E.; PUY, D. Has globalization really increased business cycle synchronization? Washington: International Monetary Fund, 2016. 
MONTINARI, L.; STRACCA, L. Trade, finance or policies: what drives the crossborder spill-over of business cycles? Journal of Macroeconomics, v. 49, n. 3, p. 131-148, 2016.

RUA, A. Measuring comovement in the time-frequency space. Journal of Macroeconomics, v. 32, n. 2, p. 685-691, 2010.

SANTOS, B. O ciclo econômico da América Latina dos últimos 12 anos em uma expectativa de restrição externa. Revista do BNDES, Rio de Janeiro, n. 43, p. 205$251,2015$.

SOARES, M.; AGUIAR-CONRARIA, L. Business cycle synchronization and the Euro: a wavelet analysis. Journal of Macroeconomics, v. 33, n. 3, p. 477-489, 2011.

STOCK, J.; WATSON, M. Introduction to econometrics. Boston: Addison Wesley, 2003.

TSE, Y.; TSUI, A. A multivariate generalized autoregressive conditional heteroscedasticity model with time-varying correlations. Journal of Business \& Economic Statistics, v. 20, n. 3, p. 351-362, 2002.

WOOLDRIDGE, J. Introdução à econometria: uma abordagem moderna. São Paulo: Pioneira Thomson Learning, 2010. 